\title{
Clinical outcome of FLAG-IDA chemotherapy sequential with Flu-Bu3 conditioning regimen in patients with refractory AML: a parallel study from Shanghai Institute of Hematology and Institut Paoli-Calmettes
}

\author{
Ling Wang ${ }^{1} \cdot$ Raynier Devillier ${ }^{2} \cdot$ Ming Wan $^{3} \cdot$ Justine Decroocq $^{2} \cdot$ Liang Tian $^{3} \cdot$ Sabine Fürst $^{2} \cdot$ Li-Ning Wang $^{1}$. \\ Norbert $\mathrm{Vey}^{2} \cdot$ Xing Fan ${ }^{1} \cdot$ Didier Blaise $\mathbb{1}^{2} \cdot$ Jiong $\mathrm{Hu}^{1}$
}

Received: 23 January 2018 / Revised: 28 June 2018 / Accepted: 30 June 2018 / Published online: 6 August 2018

(c) The Author(s) 2018. This article is published with open access

\begin{abstract}
The purpose of the study was to evaluate the feasibility of conditioning regimen with sequential chemotherapy (FLAG-IDA), followed by Fludarabine (5 days) + Busulfan ( 3 days) by parallel analysis of patients with refractory acute myeloid leukemia (AML) from two transplantation centers in China and France. A total of 47 refractory AML with median bone marrow blast of 35\% (1-90\%) and median age at 42 years (16-62) were enrolled. Thirteen patients received peripheral stem cell transplantation (HSCT) from HLA-matched sibling donor, while 18 and 16 from unrelated or haplo-identical donors, respectively. With a median follow-up of 24.3 months (1-70), 13 patients relapsed at a median time of 5.1 months (2.2-18.0) and 24 patients died due to relapse $(n=12)$ or non-relapsed mortality (NRM, $n=12)$. The estimated 3-year RR and NRM were $33.5 \pm 5.7 \%$ and $25.7 \pm 4.2 \%$, respectively. The estimated 3-year overall survival (OS) and event-free survival (EFS) were $43.8 \pm 7.8 \%$ and $42.3 \pm 7.8 \%$. In multivariate analysis, age $(<40)$ and low bone marrow blast were associated with better EFS, while no difference was observed between the two centers. The patients enrolled in study were unselected, representing typical patients' population of refractory AML, and primary data demonstrated the feasibility of sequential conditioning regimen.
\end{abstract}

These authors contributed equally: Ling Wang, Raynier Devillier.

These authors jointly supervised this work: Didier Blaise, Jiong Hu.

Didier Blaise

BLAISED@ipc.unicancer.fr

$\triangle$ Jiong $\mathrm{Hu}$

hj10709@rjh.com.cn

1 Shanghai Institute of Hematology, Department of Hematology, Blood and Marrow Transplantation Center, Collaborative Innovation Center of Hematology, Rui Jin Hospital, Shanghai Jiao Tong University School of Medicine, 18F/OPD Bldg 197 Rui Jin Road II, 200025 Shanghai, China

2 Department of Hematology, Program of transplantation and cell therapy, Program of leukemia, Centre de recherche en Cancérologie de Marseille (CRCM), Institut Paoli-Calmettes, Aix Marseille University, 232 Boulevard Sainte Marguerite, 13273, Marseille CEDEX 9, France

3 Shanghai Clinical Research Center (SCRC), (Feng Lin International Centre), 18F Bldg A, 380 Feng Lin Road, 200032 Shanghai, China

\section{Introduction}

The prognosis of patients with refractory acute myeloid leukemia (AML) is poor. Primary treatment failure or primary refractory AML is usually defined by failure to achieve 50\% reduction in blast numbers or achieve a complete hematologic remission (CR) after two courses of induction chemotherapy, while refractory-relapsed patients were defined as failure to obtain CR after relapse [1-3]. For these patients, the overall response to salvage the chemotherapy of high-dose cytosine arabinoside (Ara-C)-based regimen, including combination of anthracycline, fludarabine, or gemtuzumab-ozogamicin, remained $<30-50 \%$, and most patients eventually relapsed within 3-6 months, even though, clinical remission (CR) can be achieved [4, 5]. Allogeneic stem cell transplantation (alloHSCT) is considered as the only curative therapy for patients with refractory AML. Based on the conventional conditioning, high relapse rate (RR) and/or non-relapse mortality (NRM) lead to dismal overall survival (OS) as low as $10-20 \%[6,7]$. 
To tackle the problem, sequential transplant approach was developed, which combines intensive salvage chemotherapy to decrease the leukemia cell burden with reduced intensive conditioning regimen (RIC). The first report of sequential FLAMSA strategy (fludarabine, intermediate dose Ara-C, and amsacrine followed by $4 \mathrm{~Gy}$ total body irradiation, cyclophosphamide, and anti-thymocyte globulin (ATG)) achieved an improved OS and leukemiafree survival (LFS), particularly for patients with only 1-2 cycles of chemotherapy before transplantation. However, for patients who received three or more cycles of chemotherapy, the overall outcome remained unsatisfactory in terms of high RR and NRM [8]. We developed a new transplantation protocol with a salvage chemotherapy composed of granulocyte colony-stimulating factor (GCSF), fludarabine, Ara-C, and idarubicin (FLAG-IDA) given before transplantation. With a 7-day interval, reduced-intensity conditioning with fludarabine and 3-day busulfan (Flu-Bu3) were given usually at the hematological nadir of previous FLAG-IDA chemotherapy. In our previous phase II clinical study, we tested the feasibility and outcome of this regimen in patients with refractory AML and demonstrated a significantly reduced relapse and improved OS compared with allo-HSCT with conventional conditioning regimen [9].

Based on this intense transplantation protocol, the feasibility of the strategy remains to be determined. We are particularly interested to compare the real practice of refractory AML treatment in two centers in terms of patients' characteristics (median age and GVHD prophylaxis strategy) and to confirm the feasibility of this transplantation strategy. Thus the study was designed to compare the overall outcome between the two centers and then to confirm the feasibility of this sequential transplantation approach in refractory AMLs. These parallel studies were developed at Rui Jin hospital (RJH, Shanghai, China) and Institut Paoli-Calmettes (IPC, Marseille, France) for patients with refractory AML to evaluate the overall transplantation outcome and confirm the feasibility of the sequential regimen, combining FLAG-IDA, before allo-HSCT, with Flu-Bu3 regimen in a heavily treated, but relatively young patients population from $\mathrm{RJH}$ and also in a more elderly, although less heavily treated patients' series (IPC) with PTCY as GVHD prophylaxis.

\section{Patients and methods}

\section{Study design and inclusion criteria}

This retrospective study included a total of 47 patients diagnosed with refractory AML who underwent allo-HSCT between 2011 and 2016. The study was approved by the two participating center's institutional review board. Informed consent from patients and donors was obtained. Patients aged between 16 and 60 years were eligible for the study if they had a refractory AML defined by either: (i) primary induction failure (PIF) with less than $50 \%$ reduction in bone marrow blast, with $>15 \%$ residual blasts after one cycle of induction chemotherapy, or persistence of $>5 \%$ leukemic blasts in the bone marrow after two induction chemotherapy, or persisting hypoplasia defined by a hypocellular bone marrow and incomplete reconstitution of cell counts in the peripheral blood (absolute neutrophil count $<0.5 \times 10^{9} / \mathrm{L}$ or platelet count $<50 \times 10^{9} / \mathrm{L}$ ) at day 100 after starting chemotherapy; (ii) early relapse, with the duration of first remission $<6$ months; (iii) relapse disease refractory to at least one cycle of salvage chemotherapy containing high-dose Ara-C; or (iv) patients with multiple relapses. Induction and re-induction chemotherapy were given according to the two institutions' preference. All donor/recipient pairs were typed at the allelic level (HLA-A, HLA-B, HLA-Cw, HLA-DRB1, and HLA-DQB1). Transplantation was performed in case of either fully matched sibling donor (MSD) or 9-10 out of 10 matched unrelated stem cell donors (match, MUD) or haploidentical-related donors (Haplo). Exclusion criteria were patients with active leukemic infiltration of the central nervous system, AML with M3 subtype, serum creatinine above $1.0 \mathrm{mg} / \mathrm{dL}$, creatinine clearance less than $60 \mathrm{~mL} / \mathrm{min}$, bilirubin above $1.5 \mathrm{mg} / \mathrm{dL}$, aminotransferases or alkaline phosphatase above 2.5 times the upper normal limit, acute or chronic heart failure, and pregnancy.

\section{Treatment}

Patients included in the study proceeded directly to the sequential cytoreductive chemotherapy followed by alloHSCT. The chemotherapy regimen consisted of $30 \mathrm{mg} / \mathrm{m}^{2} /$ day fludarabine and $1 \mathrm{~g} / \mathrm{m}^{2} /$ day Ara-C for 5 consecutive days (day-21 to day-17) and idarubicin $12 \mathrm{mg} / \mathrm{m}^{2} /$ day (RJH) or $10 \mathrm{mg} / \mathrm{m}^{2} /$ day (IPC) for 3 days (day-17 to day-15). No other chemotherapy was given within 3 weeks of FLAGIDA chemotherapy, except for hydroxyurea in case of hyperleukocytosis. Seven days after chemotherapy, preparative regimen was given with $30 \mathrm{mg} / \mathrm{m}^{2} /$ day fludarabine for 5 consecutive days (days -7 to -3 ) and $3.2 \mathrm{mg} / \mathrm{kg} /$ day i.v. busulfan for 3 consecutive days (days -5 and -3 ) [9]. Day 0 was designated as the day of graft infusion with G-CSF-mobilized peripheral blood stem cell for all patients. There were two different prophylaxis strategy for acute graft versus host disease (aGVHD). In RJH, the regimen included standard cyclosporine with short-course methotrexate (MTX) and mycophenolate mofetil (MMF) for MSD, while additional ATG for 4 consecutive days (days -4 to -1 ) was given in case of MUD (1.5 mg/kg/day) or Haplo $(2.5 \mathrm{mg}$ / 


\begin{tabular}{lllll}
\hline & Total & RJH & IPC & P \\
\hline No. of patients & 47 & 27 & 20 & 0.22 \\
Sex: Male/female & $17 / 30$ & $11 / 16$ & $6 / 14$ & 0.18 \\
Age: median (range) & $42(16-62)$ & $34(16-60)$ & $44(26-62)$ & 0.001 \\
Disease stage & & & & \\
Induction failure/early relapse & $23 / 1$ & $14 / 1$ & $9 / 0$ & 0.47 \\
Relapse/refractory & 23 & 12 & 11 & \\
Cytogenetic/molecular & & & & 0.045 \\
Favorable/intermediate/poor/not evaluable & $2 / 18 / 19 / 8$ & $1 / 9 / 9 / 8$ & $1 / 9 / 10 / 0$ & \\
Previous chemotherapy median (range) & $3(1-10)$ & $4(2-10)$ & $2(1-3)$ & $<0.001$ \\
WBC (x109/L) & 3.0 & 4.4 & 3.0 & 0.27 \\
Median (range) & $(0.3-44)$ & $(0.3-11.1)$ & $(0.7-28.0)$ & \\
Circulating blast (positive/negative) & $28 / 19$ & $17 / 10$ & $11 / 9$ & 0.58 \\
BM blast: median (range) & $35 \%(1-90)$ & $56 \%(1-90)$ & $17.5 \%(4-79)$ & 0.01 \\
Donor type & & & & \\
MSD/MUD/Haplo & $13 / 16 / 16$ & $8 / 11 / 8$ & $5 / 7 / 8$ & 0.76 \\
aGvHD prophylaxis & & & & $<0.001$ \\
CSA + MTX + MMF \pm ATG/PT-Cy + CSA \pm MMF & $27 / 20$ & $27 / 0$ & $0 / 20$ & $<0.001$ \\
DLI post HSCT (yes/no) & $13 / 34$ & $2 / 25$ & $11 / 9$ & \\
\hline RJH Rui Jin Hospital, & & & \\
\end{tabular}

$R J H$ Rui Jin Hospital, IPC Institut Paoli-Calmettes, $B M$ bone marrow, $M S D$ matched sibling donor, $M U D$ matched unrelated donor, Haplo haplo-identical donor, $a G v H D$ acute graft-versus-host disease, CsA cyclosporine A, MTX methotrexate, MMF mycophenolate mofetil, ATG anti-thymocyte globulin, $P T-C y$ posttransplant cyclophosphamide, DLI donor lymphocyte infusion

$\mathrm{kg} /$ day, Table 1). As to the GVHD prophylaxis at IPC, all patients received $50 \mathrm{mg} / \mathrm{kg}$ cyclophosphamide (PT-Cy) (day +3 and +4 ) and cyclosporine started on day +5 alone (for MSD and 10/10 MUD) or in combination with MMF in case of 9/10 MUD or Haplo. There were also two strategies for donor lymphocyte infusion (DLI). In RJH, DLI was given in case of loss of donor chimerism, persistent, or increased minimal residual disease documented. In IPC, prophylaxis DLI (pDLI) was given for all patients without active GVHD as shown in Table 1.

\section{Clinical outcomes, toxicity, and GVHD assessment}

The study endpoints included engraftment, clinical response after transplantation, OS, event-free survival (EFS), relapse, non-relapsed mortality (NRM), acute GVHD, and chronic GVHD. Time to neutrophil recovery was defined as the first of 3 consecutive days in which the absolute neutrophil count exceeded $0.5 \times 10^{9} / \mathrm{L}$ and engraftment failure as an absolute neutrophil count was above $0.5 \times 10^{9} / \mathrm{L}$ at day +42 after allo-HSCT. Leukemia response rate was evaluated at day $+30,+60$, and +90 and $6,12,18$, and 24 months after the transplant. The electronic medical record was reviewed to grade toxicities according to the National Cancer Center Common Toxicity Criteria (NCI-CTC) Version 2.0. GVHD was evaluated according to the Seattle standard criteria [10].

\section{Statistical methods}

All data were collected from patients' chart with last followup at March 31, 2017. All data was locked in April 2017. The statistical analysis was performed in the Shanghai Clinical Research Center (SCRC). OS and EFS were calculated using the Kaplan-Meier method. OS was defined as the time from allo-HSCT to death, regardless of the cause. EFS was defined as survival without event, such as no clinical response after transplantation, disease relapse, or death due to NRM. The probability of NRM and relapse were analyzed with cumulative incidence competing risk (CICR). We defined NRM as death with no evidence of leukemia relapse or progression. Univariate analysis of potential prognostic factor associated with OS, EFS, and RR and NRM were performed by log-rank test and Gray's test. For multivariate analysis, the Cox models and the logistic regression model were built by testing the following covariates: treatment center (RJH versus IPC), donor type (MSD and MUD versus Haplo), patient age ( $<40$ years versus $\geq 40$ years), number of pre-transplantation chemotherapy ( $<3$ cycles versus $\geq 3$ cycles), disease stage (primary refractory versus relapse/refractory), and bone marrow blast $(<35 \%$ versus $\geq 35 \%)$. The Cox models always kept the transplantation center as a variable even if its coefficients were not significant. Other covariates were selected by the backward elimination method and were held 
in the Cox models if the $p$-value was $<0.05$. SPSS (SPSS Inc., Chicago, IL) and R version 3.4.1 software packages were used for data analysis [11].

\section{Results}

\section{Patient and donor characteristics}

The characteristics of the patients and the donors are summarized in Table 1 . The median age of recipients was 42 years (range, 16-62). AML cytogenetic status classified according to the European Leukemia Net was favorable in two patients $(4.3 \%)$, intermediate in $18(38.3 \%)$, adverse in $19(40.4 \%)$, and $8(17 \%)$ not evaluable. A total of 22 patients $(46.9 \%)$ had induction failure, $24(51.0 \%)$ with relapse/refractory disease, and only 1 patient $(2.1 \%)$ with early relapse disease during consolidation chemotherapy. The median marrow blast percentage was 35\% (1-90\%), while only 3 patients had $<5 \%$ leukemic blasts in the bone marrow. The median peripheral white blood count (WBC) was $3.0 \times 10^{9} / \mathrm{L}(0.3-44.0)$, while 28 patients had circulating blast. The donors were HLA-matched sibling (MSD, $n=13,27.7 \%$ ), and unrelated (MUD, $n=18,38.3 \%$ ) and haplo-identical siblings (haplo, $n=16,34.0 \%$ ).

\section{Engraftment}

All patients developed pancytopenia after the sequential conditioning regimen. Forty-three engrafted and four died in aplasia. The median time to neutrophil recovery $(>0.5 \times$ $10^{9} / \mathrm{L}$ ) was 17 days (range, 13-56). A total of 35 patients had platelet recovery $\left(>20 \times 10^{9} / \mathrm{L}\right)$ with a median time of 21 days (range, 12-60), while eight patients failed to obtain platelet recovery.

\section{Disease response and outcome}

At day $+28,43$ patients were evaluable for response and 42 patients achieved bone marrow remission, including 32 with complete remission (CR) and 10 without platelet recovery (CRp), while only one patient failed to obtain bone marrow remission. Thus, the overall remission rate was $89 \%(95 \%$ confidence interval: 76-96\%).

With a median follow-up of 24.3 months (range, 1.5-70.0), 13 patients relapsed with estimated 3-year accumulated relapse incidence at $33.5 \pm 5.7 \%$ (Fig. 1). The median time to relapse was 5.1 months (2.2-18.0) after transplantation. Of note, most relapse events occurred within first 6 months and only three patients relapsed beyond 6 months (at $6.5,12$, and 18, respectively) after transplantation. A total of 12 patients died due to NRM, with 3-year estimated NRM at $25.7 \pm 4.2 \%$ (Fig. 1).

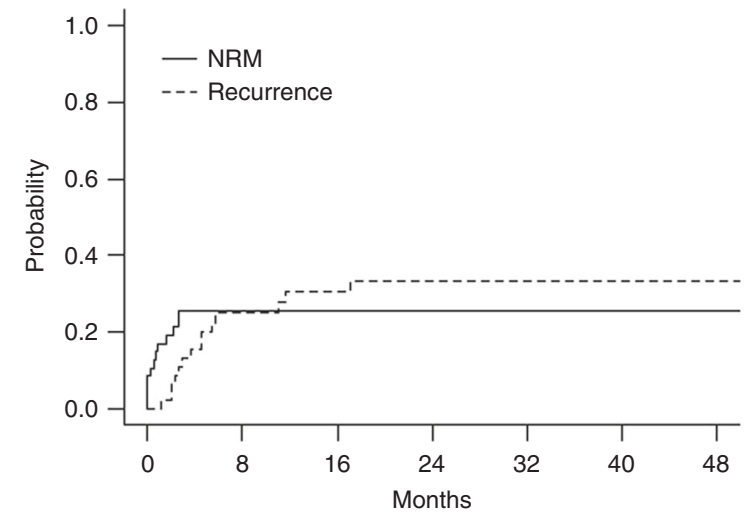

Fig. 1 Cumulative incidence of relapse and NRM for all patients
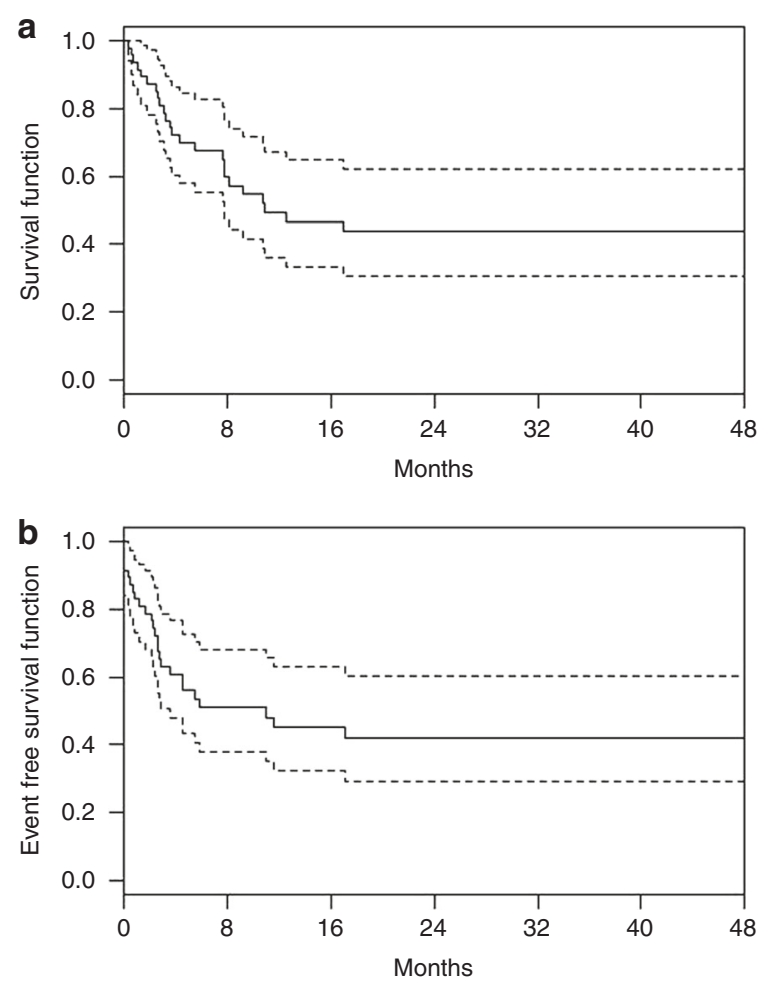

Fig. 2 Kaplan-Meier curves with $95 \%$ confidence bound for overall survival (a) and event-free survival (b)

Overall, of the 47 patients included in the study, 24 died eventually either due to disease relapse $(n=12)$ or NRM $(n=12)$, with median survival of 10.9 months for all patients. The Kaplan-Meier estimates of OS and EFS at 3 years were $43.8 \pm 7.8 \%$ and $42.3 \pm 7.8 \%$ (Fig. 2).

\section{Effect of treatment centers on transplantation outcome}

Since the patients series from the participant centers varied significantly in age, pre-transplantation chemotherapy, bone marrow blasts, GVHD prophylaxis regimen (Standard 
Table 2 Comparison of transplantation outcome between the treatment centers

\begin{tabular}{llll}
\hline & RJH $(n=27)(\%)$ & IPC $(n=20)(\%)$ & $p$-Value \\
\hline OS & $38.0 \pm 9.7$ & $52.5 \pm 12.7$ & 0.22 \\
EFS & $38.0 \pm 9.7$ & $47.8 \pm 13.0$ & 0.35 \\
NRM & $34.0 \pm 9.2$ & $15.0 \pm 8.0$ & 0.12 \\
Relapse & $28.5 \pm 12.2$ & $37.5 \pm 14.3$ & 0.74 \\
\hline
\end{tabular}

RJH Rui Jin Hospital, IPC Institut Paoli-Calmettes, OS overall survival, $E F S$ event-free survival, $N R M$ non-relapsed mortality

Table 3 Univariate and multivariate analysis for risk factors for EFS

\begin{tabular}{llll}
\hline Valuables & $\begin{array}{l}\text { Univariate } \\
\text { analysis } \\
p \text {-Value }\end{array}$ & \multicolumn{2}{l}{ Multivariate analysis } \\
\cline { 3 - 4 } & Hazard ratio & $p$-Value \\
\hline Disease status & 0.724 & - & - \\
Treatment center & 0.411 & - & - \\
$\begin{array}{l}\text { Cycles of } \\
\text { chemotherapy }\end{array}$ & 0.608 & - & - \\
Donor type & 0.122 & - & - \\
Bone marrow blast & 0.070 & $6.679(1.381$, & 0.018 \\
& & $32.305)$ & \\
Age & 0.143 & $1.036(1.003$, & 0.031 \\
& & $1.069)$ & \\
\hline
\end{tabular}

CsA-based regimen versus PT-Cy-based regimen), and pDLI post-transplantation as shown in Table 1 , we compared the overall transplantation outcome between the two centers (RJH versus IPC). Of note, the OS, EFS, NRM, and RR were comparable between RJH and IPC, thus confirming the efficacy and feasibility of the transplantation protocol consisting of sequential intensive chemotherapy and reduced intensity conditioning regimen as reported previously, and also demonstrated that similar outcome can be achieved in more elderly, but less pre-treated with different GVHD prophylaxis regimens (Table 2). The effect of treatment centers on EFS was further ruled out in multivariate analysis as shown in Table 3.

\section{Univariate and multivariate analysis of risk factors for transplantation outcome}

To evaluate the potential factor contribution to the transplantation outcome, further univariate and multivariate analyses were performed. Patients with marrow blast over median level $(\geq 35 \%)$ and age over 40 tended to have inferior EFS ( $p=0.070$ and 0.082 , respectively, in univariate analysis), while disease status (primary refractory versus refractory/refractory disease), cycles of previous chemotherapy, donor type, and treatment center were not significantly associated with EFS. In the multivariate analysis of risk factors associated with EFS, age over 40 and
Table 4 Univariate and multivariate analysis for risk factors for relapse

\begin{tabular}{llll}
\hline Valuables & $\begin{array}{l}\text { Univariate } \\
\text { analysis } \\
p \text {-Value }\end{array}$ & Multivariate analysis \\
\cline { 3 - 4 } & Hazard ratio & $p$-Value \\
\hline Disease status & 0.887 & - & - \\
Treatment center & 0.684 & - & - \\
Cycles of chemo & 0.640 & - & - \\
Donor type & 0.255 & - & - \\
Bone marrow & 0.314 & $6.88(0.741$, & 0.090 \\
blast & & $63.899)$ & \\
Age & 0.032 & $1.06(1.013$, & 0.012 \\
& & $1.109)$ & \\
\hline
\end{tabular}

bone marrow blast over $35 \%$ were associated with inferior EFS, as shown in Table 3.

As to the NRM, there was no associated risk factor identified via both univariate and multivariate analysis. For disease relapse, the only associated risk factor was age over 40 in both univariate and multivariate analysis, while all other factors were not relevant statistically, as shown in Table 4.

\section{Acute and chronic graft-versus-host-disease}

Among 44 patients evaluable for aGVHD, a total of 15 patients had documented II-IV aGVHD, while five had III-IV aGVHD. In 34 evaluable patients for cGVHD, 15 had cGVHD, while 10 had extensive cGVHD. Based on different median age of patients, GVHD prophylaxis, and pDLI strategy between RJH and IPC, we further evaluated the potential risk of GVHD in two cohorts and no significant difference was documented. For extensive cGVHD, we observe only one out of patients with PT-CY as GVHD prophylaxis, while six out of 17 patients with non-PT-CY strategy $(p=0.07)$.

\section{Discussion}

Allo-HSCT remains as the only curative therapy for patients with relapsed AML [5]. Though transplantation with conventional conditioning regimen is not encouraging with OS less than $20 \%$ for patients with PIF or after relapse [7, 12-14], the sequential transplant approach combining a short course of intensive chemotherapy followed by RIC conditioning had been evaluated and reported in several studies. In the first reported FLASM-RIC study, patients having received only two previous chemotherapy courses presented a 2-year NRM incidence of $22.2 \%$ and 2-year OS and LFD around $60 \%$, though more heavily pre-treated patients do poorly even after transplantation with 2-year OS 
and LFS less than 25\% [8]. In a more recent report of clofarabine-based and Ara-C-based chemotherapy followed by i.v. busulfan-based conditioning regimen in AML patients with induction failure, 2-year NRM rate was $12 \%$ with no grade IV non-hematologic adverse events. A total of $75 \%$ patients achieved CR by day +30 with 2 -year OS at $38 \%$, which was superior to the $20-30 \%$ reported after most standard allo-HSCT protocol [15]. Similarly, Middeke et al. reported a 2-year OS of $43 \%$ in relapsed/refractory AML after clofarabine salvage therapy and allogeneic SCT in patients achieving a response [16].

Our study was designed to test the efficacy and feasibility of intensive cytoreductive chemotherapy sequential with reduced-intensity conditioning regimen in patients with refractory AML. The estimated 3-year OS and EFS were $43.8 \pm 7.8 \%$ and $42.3 \pm 7.8 \%$, which confirmed the outcome in our previous pilot study. More importantly, we did not document significance of OS and EFS between two patients series from RJH and IPC by univariate and multivariate analyses, thus confirming the feasibility of our treatment protocol.

One may consider that a limitation of our study was the significant difference in the characteristics of patient's population from RJH and IPC in terms of age, pretransplantation chemotherapy, GVHD prophylaxis, and pDLI strategy. On the other hand, we were individually interested to compare the real practice of refractory AML treatment in two different countries and to test the reproducibility of the treatment outcome with FLAG cytoreductive chemotherapy, sequential with Flu-Bu conditioning, in different patient series with different transplantation systems. Though due to the number of patients enrolled in the study, which may limit the statistic power, the comparable OS and EFS and more interestingly a relatively low NRM $(\sim 15 \%)$ observed in patients treated in IPC suggested that this intense chemotherapy and conditioning protocol is feasible not only in heavily treated and younger patients (RJH), but also feasible for a more elderly although less heavily treated patients' series (IPC). Second, though it is not a study design to directly compare the standard GVHD prophylaxis to the PT-Cy-based regimen, our data support that the overall transplantation outcome in refractory AML patients was comparable and such intense treatment can be safely carried out with PT-Cy strategy, which may tend to have fewer extensive cGVHD events $[17,18]$.

In the risk factors analysis, we acknowledged that transplantation for young $(<40)$ tended to have promising EFS at $58.6 \pm 11.4 \%$ after allo-HSCT, which may suggest that in young patients with documented refractory AML, direct transplantation with sequential cytoreductive chemotherapy and reduced toxicity conditioning can be considered as an treatment option even without attempts of intensified salvage chemotherapy, which may achieve CR but usually with low response rate and accumulated toxicities. In more elderly patients $(\geq 40)$, we demonstrated that the underlying cause of treatment failure was mostly due to higher relapsed rate $(48.1 \pm 13.2 \%$ versus $20.9 \pm 10.8 \%$, $p=0.02)$ rather than NRM $(27.7 \pm 9.0 \%$ versus $23.8 \pm$ $9.3 \%, p=0.081$ ), compared with young patients. Overall, based on the survival curve of both EFS and OS reached a plateau, we may speculate that the intense sequential treatment strategy can be considered as a curative treatment for $\sim 40 \%$ refractory AML, which was at least comparable or even better than the approaches using FLASMA or clofarabine-based regimen, sequential with reduced intensity conditioning for refractory AML.

Relapse and transplantation-related toxicity remains as the two major causes of treatment failure. As to the relapse, even with such an intense protocol, still most relapse occurred within 6 months, with only three patients relapsed after 6 months, and no relapse events were documented after 2 years after transplantation. Of interest, we also observed that non-relapse death was documented mostly early after transplantation, with no NRM event after 9 months after transplantation. These observations may indicate that intensity of our treatment regimen was relatively toxic in $25 \%$ of patients, while such an intensity approach was not sufficient to control these $33 \%$ of patients with refractory AML. Simply escalation of the intensity of our sequential treatment strategy may not be able to overcome a group of patients with very refractory disease, but to increase the NRM.

As to the potential role of pDLI, with limited number of patients who actually received pDLI in our series and all were from IPC, it is difficult to evaluate its exact role when combined with our intensified transplantation protocol. But the anti-leukemia efficacy of the sequential conditioning regimen combining FLAG-IDA chemotherapy followed by Flu-Bu3 regimen may provide also an important treatment platform to further refine the post-transplantation strategies to improve disease control and prevent relapse in refractory AML, such as pDLI or maintenance therapy with hypomethylation agents $[19,20]$. Besides we speculate that new strategies such as addition of histone deacetylase inhibitor, which may potentiate the anti-leukemic effect without increased intensity of conditioning must be engaged in future optimization, particularly for high-risk patients who relapsed early even with quite intense allo-HSCT protocol [21, 22].

Though this was a study with limited number of patients, we believed that patients enrolled in this parallel analysis were unselected, representing the typical population of refractory AML, and the sequential-conditioning regimen combining FLAG-IDA chemotherapy followed by Flu-Bu3-conditioning regimen was feasible and further 
confirming clinical trial with larger patients' cohort was warranted.

\section{Compliance with ethical standards}

Conflict of interest The authors declare that they have no conflict of interest.

Open Access This article is licensed under a Creative Commons Attribution 4.0 International License, which permits use, sharing, adaptation, distribution and reproduction in any medium or format, as long as you give appropriate credit to the original author(s) and the source, provide a link to the Creative Commons license, and indicate if changes were made. The images or other third party material in this article are included in the article's Creative Commons license, unless indicated otherwise in a credit line to the material. If material is not included in the article's Creative Commons license and your intended use is not permitted by statutory regulation or exceeds the permitted use, you will need to obtain permission directly from the copyright holder. To view a copy of this license, visit http://creativecommons. org/licenses/by/4.0/.

\section{References}

1. Cheson BD, Bennett JM, Kopecky KJ, Büchner T, Willman CL, Estey $\mathrm{EH}$, et al. Revised recommendations of the International Working Group for Diagnosis, Standardization of Response Criteria, Treatment Outcomes, and Reporting Standards for Therapeutic Trials in acute myeloid leukemia. J Clin Oncol. 2003;21:4642-9.

2. Ravandi F. Primary refractory acute myeloid leukaemia - in search of better definitions and therapies. $\mathrm{Br} \mathrm{J}$ Haematol. 2011;155:413-9.

3. Ferguson P, Hills RK, Grech A, Betteridge S, Kjeldsen L, Dennis $\mathrm{M}$, et al. An operational definition of primary refractory acute myeloid leukemia allowing early identification of patients who may benefit from allogeneic stem cell transplantation. Haematologica. 2016;101:1351-8.

4. Litzow MR, Othus M, Cripe LD, Gore SD, Lazarus HM, Lee SJ, et al. Failure of three novel regimens to improve outcome for patients with relapsed or refractory acute myeloid leukaemia: a report from the Eastern Cooperative Oncology Group. Br J Haematol. 2010;148:217-25.

5. Thol F, Schlenk RF, Heuser M, Ganser A. How I treat refractory and early relapsed acute myeloid leukemia. Blood. 2015;126:319-27.

6. Oyekunle AA, Kröger N, Zabelina T, Ayuk F, Schieder H, Renges $\mathrm{H}$, et al. Allogeneic stem-cell transplantation in patients with refractory acute leukemia: a long-term follow-up. Bone Marrow Transplant. 2006;37:45-50.

7. Duval M, Klein JP, He W, Cahn JY, Cairo M, Camitta BM, et al. Hematopoietic stem-cell transplantation for acute leukemia in relapse or primary induction failure. J Clin Oncol. 2010;28:3730-8.

8. Schmid C, Schleuning M, Schwerdtfeger R, Hertenstein B, Mischak-Weissinger E, Bunjes D, et al. Long-term survival in refractory acute myeloid leukemia after sequential treatment with chemotherapy and reduced-intensity conditioning for allogeneic stem cell transplantation. Blood. 2006;108:1092-9.
9. Tang W, Fan X, Wang L, Hu J, Busulfan and fludarabine conditioning regimen given at hematological nadir of cytoreduction fludarabine, cytarabine, and idarubicin chemotherapy in patients with refractory acute myeloid leukemia undergoing allogeneic stem cell transplantation: a Single Arm Pilot Consort Study. Medicine (Baltimore). 2015;94:e706

10. Przepiorka D, Weisdorf D, Martin P, Klingemann HG, Beatty P, Hows, et al. 1994 Consensus conference on acute GVHD grading. Bone Marrow Transplant. 1995;15:825-8.

11. Kanda Y. Investigation of the freely available easy-to-use software 'EZR' for medical statistics. Bone Marrow Transplant. 2013;48:452-8.

12. Fung HC, Stein A, Slovak MI, O'donnell MR, Snyder DS, Cohen $\mathrm{S}$, et al. A long- term follow-up report on allogeneic stem cell transplantation for patients with primary refractory acute myelogenous leukemia: impact of cytogenetic characteristics on transplantation outcome. Biol Blood Marrow Transplant. 2003;9:766-71.

13. Singhal S, Powles R, Henslee-Downey PJ, Chiang KY, Treleaven J, Godder K, et al. Allogeneic transplantation from HLA-matched sibling or partially HLA-mis-matched related donors for primary refractory acute leukemia. Bone Marrow Transplant. 2002;29: 291-5.

14. Biggs JC, Horowitz MM, Gale RP, Ash RC, Atkinson K, Helbig $\mathrm{W}$, et al. Bone marrow transplants may cure patients with acute leukemia never achieving remission with chemotherapy. Blood. 1992;80:1090-3.

15. Mohty M, Malard F, Blaise D, Milpied N, Socié G, Huynh A, et al. Sequential regimen of clofarabine, cytosine arabinoside and reduced-intensity conditioned transplantation for primary refractory acute myeloid leukemia. Haematologica. 2017;102:184-91.

16. Middeke JM, Herbst R, Parmentier S, Bug G, Hänel M, Stuhler G, et al. Clofarabine salvage therapy before allogeneic hematopoietic stem cell transplantation in patients with relapsed or refractory AML: results of the BRIDGE trial. Leukemia. 2016;30:261-7.

17. Luznik L, Fuchs EJ. High-dose, post-transplantation cyclophosphamide to promote graft-host tolerance after allogeneic hematopoietic stem cell transplantation. Immunol Res. 2010;47:65-77.

18. Devillier R, Granata A, Fürst S, Harbi S, Faucher C, Weiller PJ, et al. Low incidence of chronic GVHD after HLA-haploidentical peripheral blood stem cell transplantation with posttransplantation cyclophosphamide in older patients. Br J Haematol. 2017;176:131-3.

19. Goodyear OC, Dennis M, Jilani NY, Loke J, Siddique S, Ryan G, et al. Azacitidine augments expansion of regulatory $\mathrm{T}$ cells after allogeneic stem cell transplantation in patients with acute myeloid leukemia (AML). Blood. 2012;119:3361-9.

20. Liga M, Triantafyllou E, Tiniakou M, Lambropoulou P, Karakantza M, Zoumbos NC, et al. High alloreactivity of lowdose prophylactic donor lymphocyte infusion in patients with acute leukemia undergoing allogeneic hematopoietic cell transplantation with an alemtuzumab-containing conditioning regimen. Biol Blood Marrow Transplant. 2013;19:75-81.

21. Song G, Valdez BC, Li Y, Dominguez JR, Corn P, Champlin RE, et al. The histone deacetylase inhibitor SAHA sensitizes acute myeloid leukemia cells to a combination of nucleoside analogs and the DNA-alkylating agent busulfan. Leuk Lymphoma. 2014;55:1625-34.

22. Long J, Fang WY, Chang L, Gao WH, Shen Y, Jia MY, et al. Targeting HDAC3, new partner protein of AKT in the reversal of chemo-resistance in acute myeloid leukemia via DNA damage response. Leukemia. 2017;31:2761-70. 